Fetal Diagnosis and Therapy
Fetal Diagn Ther 2016;39:56-63

DOI: $10.1159 / 000381914$
Received: March 27, 2015

Accepted after revision: March 27, 2015

Published online: August 28, 2015

\title{
Placental Protein 13 Administration to Pregnant Rats Lowers Blood Pressure and Augments Fetal Growth and Venous Remodeling
}

\author{
Sveinbjorn Gizurarson ${ }^{a}$ Elisabet Run Sigurdardottir ${ }^{a}$ Hamutal Meiri ${ }^{b, c}$ \\ Berthold Huppertz $^{g}$ Marei Sammar ${ }^{d}$ Adi Sharabi-Nov ${ }^{\mathrm{e}}{ }^{\mathrm{f}}$ Maurizio Mandaláh \\ George Osoli \\ ${ }^{a}$ Faculty of Pharmaceutical Sciences, School of Health Science, University of Iceland, Reykjavik, Iceland; ${ }^{\text {b Telemarpe }}$ \\ Ltd., Tel Aviv, ' ${ }^{\mathrm{H}} \mathrm{H}$ Laboratories Ltd., Rehovot, ${ }^{\mathrm{d}}$ Department of Biotechnology Engineering, ORT Braude College, \\ Karmiel, ${ }^{2}$ Ziv Medical Center, Safed, and ${ }^{\mathrm{f}}$ Tel Hai College, Tel Hai, Israel; ' Institute of Cell Biology, Histology

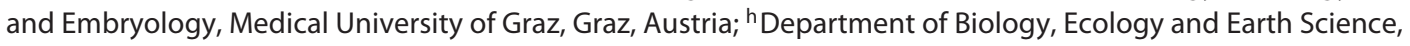 \\ University of Calabria, Arcavacata di Rende, Italy; 'Department of Obstetrics, Gynecology and Reproductive \\ Sciences, University of Vermont College of Medicine, Burlington, Vt., USA
}

\section{Key Words}

Placental protein $13 \cdot$ Angiogenesis $\cdot$ Animal model $\cdot$ Fetal growth · Fetal therapy · First trimester · Placenta

\begin{abstract}
Reduced first-trimester concentrations of placental protein 13 (PP13) are associated with subsequent development of preeclampsia, a major pregnancy disorder. We previously showed that PP13 has a vasodilatory effect, reduces blood pressure and augments expansive remodeling of the uteroplacental vasculature in pregnant rats. In this study, slowrelease osmotic pumps were implanted in gravid rats (on day 8) to provide 1 week of PP13 supplementation. Treatment was associated with a reversible blood pressure reduction that returned to normal on day 15 . In addition, PP13 caused venous expansion that is larger in the venous branches closer to the placenta. Then, it increased placental and pup weights. Similar administration of a truncated PP13 variant (DelT $T_{221}$ ) that is unable to bind carbohydrates (a rare spontaneous mutation associated with a high frequency of severe early preeclampsia among Blacks in South Africa) produced
\end{abstract}

\begin{tabular}{|c|c|c|}
\hline \multirow{3}{*}{$\begin{array}{l}\text { KARGER } \\
\text { E-Mail karger@karger.com } \\
\text { www.karger.com/fdt }\end{array}$} & (C) 2015 The Author(s) & \multirow{3}{*}{$\begin{array}{l}\text { Karger } \\
\text { Open access }\end{array}$} \\
\hline & Published by S. Karger & \\
\hline & & \\
\hline www.karger.com/fdt & $\begin{array}{l}\text { This article is licensed under the Creativ } \\
\text { NonCommercial-NoDerivatives } 4.0 \text { Inter } \\
\text { NC-ND) (http://www.karger.com/Servi } \\
\text { Usage and distribution for commercial pu } \\
\text { tribution of modified material requires wr }\end{array}$ & $\begin{array}{l}\text { Commons Attribution- } \\
\text { tional License (CC BY- } \\
\text { s/OpenAccessLicense). } \\
\text { poses as well as any dis- } \\
\text { ten permission. }\end{array}$ \\
\hline
\end{tabular}
tribution of modified material requires written permission. a hypotensive effect similar to the full-length molecule, but without venous remodeling and increased placental and pup weights. These results indicate the importance of PP13 carbohydrate binding for inducing vascular remodeling and improving reproductive outcome. Future studies are needed to determine whether beneficial effects would be evident in animal models of preeclampsia or in women predisposed to the development of preeclampsia.

\section{(C) 2015 The Author(s)}

Published by S. Karger AG, Basel

\section{Introduction}

Preeclampsia is a major cause of maternal, fetal and neonatal morbidity worldwide. This condition affects $2-8 \%$ of pregnancies $[1,2]$, and the etiology and pathophysiology are still not fully understood. There is evidence for multifactorial causes, although the origin of this syndrome is thought to develop very early during placental development, possibly due to placental dysfunctional growth [3]. Therapeutic options are limited and include: (1) administration of aspirin in the first trimester to pre-

Sveinbjorn Gizurarson

Faculty of Pharmaceutical Sciences University of Iceland

Hofsvallagata 53, IS-107 Reykjavik (Iceland) E-Mail sveinbj@hi.is 
vent the development of the disorder [4] or (2) conservative use of antihypertensive drugs at the time of the disease [5].

Several new experimental protocols are being developed to treat preeclampsia: (1) apheresis to remove soluble fms-like tyrosine kinase 1 (sFlt-1), the circulating receptor for vascular endothelial growth factor and placental growth factor [6], (2) injection of placentally derived stem cells to reduce blood pressure and ameliorate endothelial damage $[7,8]$, or (3) replenishing pregnant women with a hydrogen sulfide donor to block the release of sFlt-1 and soluble endoglin [9]. To date, none of these procedures have been evaluated by a randomized clinical trial or as a preventive strategy.

At the same time, the field of preeclampsia research has progressed significantly due to major discoveries that improve our ability to predict and stratify the risk for developing the disorder by using various biochemical and biophysical markers. For example, the blood of preeclamptic women was found to have elevated levels of subcellular fragments/necrotic particles originating from the villous syncytiotrophoblast $[10,11]$. These seem to be fairly specific to preeclampsia, since they are absent in normal pregnancy or in gestations characterized by fetal growth restriction $[12,13]$. Other potentially useful serum biomarkers, also expressed by the syncytiotrophoblast, are placental growth factor, sFlt-1, soluble endoglin, and placental protein 13 (PP13) [13, 14]. It has been estimated that developing algorithms that use several biomarkers, combined with hemodynamic measurements such as uterine artery pulsatility and resistance indices and mean arterial pressure, could lead to the prediction of $60-90 \%$ of preeclampsia cases (at a $5-10 \%$ false-positive rate) before 13 weeks of gestation [15].

PP13 is a member of the galectin family of sugar-binding proteins and is also called galectin $13[16,17]$. This protein is expressed by the placenta from the time of trophoblast fusion to form the syncytiotrophoblast layer and can be detected in maternal blood after week 5-6 of gestation $[13,20]$. Its function and pharmacokinetics are not fully understood. It has been shown that PP13 interacts with the immune system by inducing apoptosis of T cells and macrophages, and is thereby potentially important in immunomodulation at the maternal-placental interface $[8,16,18]$. A review of our current knowledge [13] has provided a meta-analysis of its clinical performance in preeclampsia risk prediction and the analysis of its naturally occurring polymorphism in pregnant women [13].

PP13 is mostly found in the multinucleated syncytiotrophoblast covering placental villi [20-22]. It is released

PP13 Administration to Pregnant Rats into the intervillous space [23], from where it enters the maternal systemic circulation [21, 23, 24]. In healthy pregnancy, serum PP13 concentrations rise moderately from the first to the third trimester [20]. However, lower first-trimester levels of maternal serum PP13 [12, 25, 26] and its encoding mRNA $[27,28]$ have been shown not only in cases developing early-onset preeclampsia, but also in those patients developing a severe form of lateonset preeclampsia $[13,20]$. PP13 has therefore been proposed as a specific biomarker for predicting preeclampsia in early pregnancy $[13,23,25,26]$.

A naturally occurring single nucleotide deletion of thymidine in position 221 is a rare mutation identified among Blacks in South Africa, which is associated with the formation of an early stop codon and the development of a shorter PP13 ('truncated' or 'DelT $\mathrm{T}_{221}$ '). This rare mutation is also associated with an elevated risk of severe (early) preeclampsia in its heterozygous version, and complete pregnancy failure with the homozygous mutation [29]. Truncated PP13 lacks the ability to induce T-cell apoptosis and immune tolerance of the decidua to extravillous trophoblast [18]. Therefore, using the truncated protein expressed by the DelT ${ }_{221}$ PP13 variant could serve as a method for better understanding the role of PP13 in preeclampsia as it provides some insight into the underlying structure-function relationships.

Our previous study on the pharmacological effects of PP13 in gravid rats [30] showed that its administration leads to a reduction in arterial blood pressure, and may induce uterine artery vasodilatation, accompanied by changes or remodeling of the uterine vasculature; both effects would favor an augmentation of uteroplacental perfusion. This study raised the question whether PP13 may be used as a biopharmaceutical to prevent preeclampsia if it were administered to women having low levels of PP13 early in the first trimester of pregnancy. Here, too, the truncated form of the mutation (i.e. DelT ${ }_{221}$ ) may be a useful tool to assess whether the carbohydratebinding domain is required for these effects.

In this study, we evaluated the hypothesis that PP13 supplementation during early pregnancy augments the process of maternal uterine vascular remodeling, specifically, the rather dramatic growth of the uterine veins noted in preliminary experiments. We also explored what of the normal PP13 effects would be mimicked by its truncated mutant, thereby substantiating the importance of vascular actions of normal PP13 during pregnancy and providing an insight into why having this rare mutation may lead to the development of severe early preeclampsia.

Fetal Diagn Ther 2016;39:56-63

DOI: $10.1159 / 000381914$ 


\section{Materials and Methods}

Animals and Approvals

The animal studies were approved by the Institutional Animal Care and Use Committee (IACUC) at the University of Vermont, Burlington, Vt., USA. All studies were carried out in accordance with the US NIH guidelines for the care and use of laboratory animals. Food and water were provided ad libitum. All efforts were undertaken according to the ' $3 \mathrm{R}$ principles' (www.nc3rs.org.uk) to reduce the number of animals used in this study and optimize experimental protocols for obtaining maximum data from each tested animal.

\section{Materials}

Recombinant wild-type PP13 and mutated PP13 were kindly prepared and provided for this project by the Hy Laboratories, Ltd. (Rehovot, Israel). The full length of wild-type PP13 was previously cloned in PQE-30 plasmid $[23,31,32]$ and was used as a template to amplify the wild-type PP13 and its DelT 221 PP13 mutant (referred to as 'truncated') sequence by PCR. Two primers were designed with the following sequences: a sense primer: CGAATCCATGTCTTCTTTACCCGTGC, and an antisense primer: TAAGTCGAGCTCCATCCATATCCCAAACTCAC. The restriction site sequences of Bam H I and Sac I were introduced in the sense and antisense primers, respectively. Both primers were synthesized by Sigma-Genosys (Rehovot, Israel). To amplify the truncated PP13 DNA sequence, 1 ng of wild-type PP13 DNA was used as a template, $0.1 \mu \mathrm{M}$ of the specific primers, $1 \mathrm{U}$ of plaque-forming unit DNA polymerase (Promega, USA), $200 \mu \mathrm{M}$ of deoxyribonucleotide triphosphate mix and plaque-forming unit DNA polymerase $\times 10$ buffer. PCR was carried out at the following temperature cycles: $94^{\circ} \mathrm{C}$ for $2 \mathrm{~min}, 94^{\circ} \mathrm{C}$ for $30 \mathrm{~s}, 60^{\circ} \mathrm{C}$ for $30 \mathrm{~s}$, and $72^{\circ} \mathrm{C}$ for $1 \mathrm{~min}$ over $35 \mathrm{cycles}$. A final extension was carried out at $72^{\circ} \mathrm{C}$ for $4 \mathrm{~min}$, and the PCR product, analyzed by agarose gel and revealing the expected size of $288 \mathrm{bp}$, was stored at $4^{\circ} \mathrm{C}$ until use.

The procedure for the expression of recombinant wild-type and truncated PP13 in Escherichia coli M15 strain host cells was performed according to the procedure of QIA express (Qiagen, USA) using the expression vector $\mathrm{PQE}-30$ as described by the manufacturer. The PCR product of PP13 DNA was purified by QIAquick PCR purification kit prior to ligation. Both the $\mathrm{PQE}-30$ and the purified PCR product DNA were digested with $\mathrm{BamH}$ I and Sac I (New England Biolabs, Ipswich, Mass., USA). The digested truncated PP13 insert was ligated into the digested PQE-30 expression vector using T4 ligase (NEB, Hitchin, UK) for $2 \mathrm{~h}$ at $22^{\circ} \mathrm{C}$. The E. coli M15 strain containing the repressor (pREP4) plasmid was transformed with the ligated product and cells were grown on ampicillin and kanamycin-selective agar. Positive clones were selected and confirmed by sequence analysis using a $\mathrm{PQE}$ sequencing primer (Qiagen, USA). The transfected E. coli cells were grown to an $A 600$ of 0.9 and isopropyl- $\beta$-D-thiogalactopyranoside was then added to a final concentration of $1 \mathrm{~mm}$. The cells were grown for an additional period of $4 \mathrm{~h}$, pelleted for $20 \mathrm{~min}$ at $4,000 \mathrm{~g}$, and stored at $-80^{\circ} \mathrm{C}$ until use. Aliquots were tested by SDS-PAGE to determine the molecular weight of recombinant proteins.

The primary structure and purity of PP13-R and of the $\mathrm{DelT}_{221}$ variant were verified by sequence analysis, molecular weight verification on SDS-PAGE revealing a molecular weight of 18 and 11 $\mathrm{kDa}$ for the wild-type and the $\mathrm{DelT}_{221}$ variant, respectively. They were further characterized by Western blotting and ELISA with polyclonal and monoclonal antibodies to PP13 [31].
Animal Experiments

Female Sprague-Dawley rats (12 weeks old, purchased from Charles River Laboratories) were allowed to acclimate in the Small Animal Facility at the University of Vermont, College of Medicine for $72 \mathrm{~h}$ prior to use. After successful impregnation by a male, $2-\mathrm{ml}$ slow-release osmotic minipumps (Alzet, Model 2 ML1) were loaded with $127 \mathrm{ng}$ of PP13 and surgically implanted subcutaneously in the periscapular region $(n=9)$ on day 8 of pregnancy. The pumps released $0.625 \mathrm{ng}$ PP13/h for 1 week. Control animals received similar osmotic pumps releasing saline $(n=6)$ or DelT 221 mutant PP13 $(\mathrm{n}=6)$, also loaded with $127 \mathrm{ng}$, to release $0.625 \mathrm{ng}$ DelT $_{221}$ mutant PP13/h.

Blood pressure and heart rate were measured prior to implantation of the osmotic pumps on day 8 and then on gestational days 10,13 and 15 using a Coda 6 tail cuff oscillometric blood pressure system (Kent Scientific Corp.). The animals were allowed to acclimatize in the measurement chambers for about $30 \mathrm{~min}$ prior to the initiation of measurement.

\section{Uterine Vasculature, Pups and Placenta}

Each animal was euthanized with an intraperitoneal injection of Nembutal on either day 15 or 21 of gestation, followed by decapitation, laparotomy, and dissection of the uterus and its vasculature. The entire uterus, including placentas and pups, was pinned in a Petri dish coated with silicone (SilGard) and filled with $10 \mathrm{mM}$ HEPES saline at $4^{\circ} \mathrm{C}$ containing a mixture of papaverine $(0.1 \mathrm{mM})$ and diltiazem $(1 \mu \mathrm{M})$ to assure complete relaxation. Each uterine horn and its vascular arcade were photographed through a calibrated stereomicroscope (Zeiss, Germany) to estimate and compare the vascular structure between groups.

The diameters of the veins were measured directly from the photos by randomly selecting and measuring 6-10 different sites in each horn, and were then averaged. The number of pixels that spanned $1 \mathrm{~mm}$ was measured and used to convert the number of pixels into millimeters, respectively. The vessels measured were: (1) main uterine vein, (2) arcuate or proximal radial veins, and (3) postplacental or premyometrial radial veins. Finally, all of the pups and placentas were dissected and weighed $(\mathrm{g})$.

\section{Statistics and Calculations}

Groups were compared by ANOVA (for large samples) or by the Kruskal-Wallis test (for small samples). The nonparametric tests were used to evaluate the significance of differences in treatment between the study groups as a whole and between any of the treatment groups (PP13 or DelT 221 ) and saline control. Values are expressed as mean \pm SD (standard deviation), with $p$ values $<0.05$ considered significant.

\section{Results}

\section{Systemic Effects of PP13 on Blood Pressure}

Figure 1 shows the changes in blood pressure prior to the insertion of osmotic pumps (gestational day 8) compared to subsequent gestational days 10,13 and 15. There was a significant (20-30\%) decrease in the mean arterial pressure in the presence of either wild-type PP13 or
58
Fetal Diagn Ther 2016;39:56-63 DOI: $10.1159 / 000381914$
Gizurarson et al. 


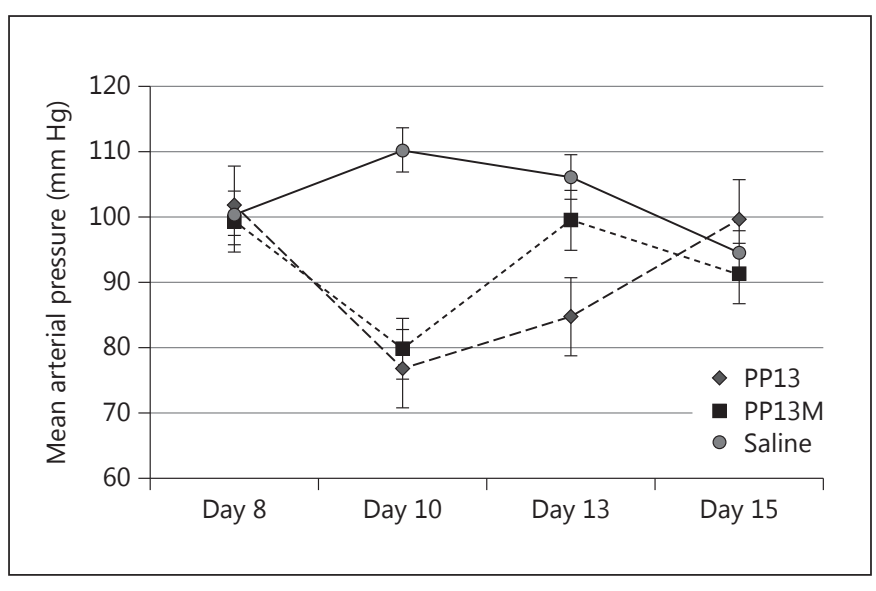

Fig. 1. Mean arterial pressure of pregnant rats after implanting osmotic pumps releasing PP13 $(n=9), \operatorname{PP} 13 M(n=6)$ or saline $(n=$ 6). Mean arterial pressure was followed over the next 7 days until gestational day 15 . Data are reported as mean \pm SD.

Table 1. Uterine vein diameters (mean $\mathrm{mm} \pm \mathrm{SD}$ ) in rats on gestational days 15 and 21 following continuous administration of PP13 or the $\operatorname{DelT}_{221}$ mutant (PP13M) from days 8 to 15 of pregnancy via subcutaneous osmotic pumps (see Materials and Methods for details)

\begin{tabular}{llll}
\hline $\begin{array}{l}\text { Gestational age and } \\
\text { type of vein }\end{array}$ & Control & PP13 & PP13M \\
\hline Day 15 & & & \\
$\quad$ Postplacental veins & $0.21 \pm 0.09$ & $0.39 \pm 0.20^{*}$ & $0.20 \pm 0.05$ \\
$\quad$ Premyometrial veins & $0.33 \pm 0.12$ & $0.41 \pm 0.13^{*}$ & $0.34 \pm 0.12$ \\
$\quad$ Radial veins & $0.32 \pm 0.11$ & $0.42 \pm 0.10^{*}$ & $0.35 \pm 0.11$ \\
Day 21 & & & \\
$\quad$ Postplacental veins & $0.28 \pm 0.07$ & $0.41 \pm 0.11^{*}$ & $0.24 \pm 0.09$ \\
$\quad$ Premyometrial veins & $0.55 \pm 0.34$ & $0.61 \pm 0.14^{*}$ & $0.33 \pm 0.16$ \\
$\quad$ Radial veins & $0.75 \pm 0.32$ & $0.91 \pm 0.32$ & $0.52 \pm 0.15$ \\
\hline
\end{tabular}

${ }^{*} \mathrm{p} \leq$ 0.05: significant differences from control values. All comparisons were made within the vein type at that particular gestational age using ANOVA followed by the Kruskal-Wallis multiple comparisons test to evaluate differences between treatment means.

DelT $_{221}$ mutant, compared to saline controls on day 10, with a progressive attenuation of this hypotensive effect on day 13. By day 15, the blood pressure-lowering effects of wild-type or mutant PP13 were no longer present.

\section{Local Effects on Uterine Vasculature}

Examination of the uterine vasculature on gestational day 15 and day 21 showed significantly larger venous di-

PP13 Administration to Pregnant Rats ameters in the group receiving wild-type PP13, compared with the saline control and DelT ${ }_{221}$ PP13 variant (table 1). Image analysis revealed that expansion was evident in all branches of the uteroplacental venous vasculature on day 15 of pregnancy, which corresponded to the end of the treatment period. By day 21 (when the osmotic pumps had been empty for 6 days), a significant residual effect was still present in the postplacental and premyometrial, but not radial veins.

Veins in the DelT $\mathrm{T}_{221}$-treated animals did not undergo the enlargement seen in the PP13-treated group at either time point, and sometimes trended to be narrower than those of saline controls, although this did not reach statistical significance, likely due to the small sample size.

\section{Pups and Placenta}

Pup Weights

On day 15, pup weights were increased in both the PP13 and the DelT $\mathrm{T}_{221}$ mutated PP13 groups relative to controls. The average number of pups was the same regardless of treatment (table 2). By day 21, pup weights were still increased in the PP13 group; however, those from the PP13 mutant group were significantly lighter than those from either the control or PP13 groups.

These differences revealed a striking difference in growth rate within the PP13 mutant group between days 15 and 21; specifically, pup weights increased about 15and 14-fold for the control (saline) and wild-type PP13 group, respectively, while the pups from the DelT $221 \mathrm{mu}$ tated PP13 group had only grown about 5.5-fold, reflecting a marked stunting of growth (table 2).

\section{Placental Weights}

Placental weights were smaller in the PP13 mutant group relative to controls or PP13 animals on day 15; by day 21 , placental weights were significantly greater in the PP13 group versus control or PP13 mutants (and the latter were significantly smaller than controls). Here, too, the rate of growth (from days 15 to 21) was altered by treatment, as the placentas in the wild-type PP13-treated group grew 3.7-fold, while the placentas in the saline and the DelT 221 groups grew only 2.5- and 2.3-fold, respectively. Hence, by day 21, the placentas were largest in the PP13-treated group and smallest in the mutant group, with intermediate values recorded in controls (table 2).

\section{Number of Pups and Pregnancy Burden}

There were no differences noted in number of pups at either time point (table 2) or in the rates of resorption (data not shown). Pregnancy burden was calculated as the

Fetal Diagn Ther 2016;39:56-63 DOI: $10.1159 / 000381914$ 
Table 2. Weights (mean \pm SD) of pups and placentas on gestational days 15 and 21 following continuous administration of PP13 or the DelT $_{221}$ mutant (PP13M) from days 8 to 15 via subcutaneous osmotic pumps (see Materials and Methods for details)

\begin{tabular}{|c|c|c|c|}
\hline $\begin{array}{l}\text { Gestational age and } \\
\text { parameter }\end{array}$ & Control & PP13 & PP13M \\
\hline \multicolumn{4}{|l|}{ Day 15} \\
\hline Pup weight, g & $0.15 \pm 0.03$ & $0.18 \pm 0.05^{\mathrm{a}}$ & $0.17 \pm 0.04^{\mathrm{b}}$ \\
\hline Placental weight, $g$ & $0.18 \pm 0.04$ & $0.17 \pm 0.04^{\mathrm{a}}$ & $0.16 \pm 0.03^{b}$ \\
\hline Number of pups & $15.5 \pm 1.0$ & $13.6 \pm 2.1$ & $14.3 \pm 3.8$ \\
\hline \multicolumn{4}{|l|}{ Day 21} \\
\hline Pup weight, g & $2.24 \pm 0.33$ & $2.46 \pm 0.35^{\mathrm{a}}$ & $0.93 \pm 0.11^{\mathrm{b}}$ \\
\hline Placenta weight, $g$ & $0.45 \pm 0.07$ & $0.64 \pm 0.11^{\mathrm{a}}$ & $0.37 \pm 0.09^{b}$ \\
\hline Number of pups & $12.5 \pm 4.9$ & $14.0 \pm 1.4$ & $13.5 \pm 0.7$ \\
\hline
\end{tabular}

The superscript letters indicate a significant $(\mathrm{p} \leq 0.05)$ difference from control values for that parameter at that gestational age. Differences between the PP13 and PP13M groups are indicated by different letters (e.g. 'b' vs. 'a' indicates that the PP13M value is different from the PP13 value, and that both are different from control values. A level of $p \leq 0.05$ was considered significant. $p$ was determined using ANOVA and the Tukey post hoc test for testing differences between the means of the study groups.

total weight of pups and placentas per rat. On day 15, the mean weights (in g per animal) were: 5.13, 4.81 and 4.60 for the saline control, wild-type and mutant PP13 groups, respectively, indicating no difference.

On day 21, the pregnancy burden was found to be (in g per animal): $33.6,43.3$ and 17.4 for the saline control, wild-type PP13 and mutated PP13 groups, respectively. The difference remained statistically indifferent (Kruskal-Wallis test) due to the relatively small number of animals.

\section{Discussion}

The current experiments were designed to extend the findings from a previous pilot study that showed that direct application of PP13 to isolated, pressurized rat uterine arteries elicited arterial vasodilation [30]. There, we also found some preliminary evidence to support a stimulatory effect on arterial remodeling secondary to a single in vivo injection in healthy nonpregnant rats starting late in pregnancy (day 13).

There were a few principal findings in the current study, in which the wild-type PP13 or the $\mathrm{DelT}_{221}$ mutant form of this molecule (PP13M) that does not bind to carbohydrates were infused into pregnant rats for a 1-week period in an earlier pregnancy period (days 8-15 of a 22day gestation): (1) both the wild-type PP13 and PP13M induced systemic hypotension that was evidenced by an approximately $20 \%$ reduction in mean arterial pressure, (2) PP13 but not PP13M significantly increased fetal weights on both day 15 and day 21, (3) PP13M infusion produced a lingering effect on the rate of fetal growth during the last 'trimester' such that PP13M fetuses were approximately $50-60 \%$ lighter on day $21,(4)$ a similar pattern of increased placental weight in the PP13 group and decreased placental weights in PP13M-treated animals was observed, and (5) PP13 but not PP13M treatment was associated with significant structural enlargement of the veins that drain the placentas and uterine corpus. Each of these findings is considered below.

Hypotension was maximal on day 10, 2 days after starting the infusion of these compounds, with comparable reductions of approximately $20 \%$ in response to either the wild-type or the truncated compound. Thereafter, blood pressure returned to normal over a period of several days such that there were no differences by day 15 of gestation. Although the mutant form of PP13 is unable to bind carbohydrates [32], this does not appear to be a requisite for inducing systemic hypotension. A former study by Burger et al. [22] has shown that PP13 is capable of causing the release of prostacyclin. The latter is known to vasodilate blood vessels. It is thus postulated that hypotension is related to this molecular feature of PP13, which apparently is located in a different molecular region than the carbohydrate-binding domain.

Moreover, the mechanisms by which this is accomplished may also be central (CNS) or peripheral (via a direct action on the arterial wall); a potential secondary mediator is also postulated. Further studies, beyond the scope of this one, would be required to understand the underlying mechanism. In this regard, it is interesting that PP13 levels tend to be lower in the first trimester in women who subsequently develop preeclampsia but normal or elevated during the third trimester, as noted in several studies $[20,26,37,38]$.

The generally stimulatory effect of PP 13 on increasing fetal and placental weights was unexpected, as the fetal weights in the healthy control group were presumably sufficient for normal pregnancy outcome. Thus, PP13 somehow facilitates fetal growth as can be seen on either days 15 and 21, respectively.

Unlike the hypotensive effect, which was developed regardless of the wild-type or the mutated PP13 variant, the impact on placental and pup weights was not observed in the PP13M group, suggesting that carbohydrate binding
60

Fetal Diagn Ther 2016;39:56-63 DOI: $10.1159 / 000381914$
Gizurarson et al. 
was required, or that it was related to the anticipated altered tertiary structure of $\mathrm{PP} 13 \mathrm{M}$ compared to the normal PP13 [32]. Furthermore, pup weights in this group were reduced by $>50 \%$ on average by day 21 of pregnancy in the PP13M group compared to the other 2 groups. With mutant PP13, fetal weights were not reduced on day 15 , when the pumps were depleted, suggesting that this compound may take time to alter the trajectory of fetal growth in a way that only becomes manifest during the latter part of pregnancy, when the fetal growth rate is maximal. There was a small but statistically significant reduction in placental weights at the earlier time point in PP13M-treated rats (about 5\%); however, this does not preclude functional abnormalities in placental hemodynamics, such as an increased intraplacental resistance, that may become more significant with time. Since uteroplacental blood flow is not thought to increase until approximately day 15 of gestation, most of the hemodynamic stress in this species would be expected to occur during the last week of pregnancy. Accordingly, in addition to the striking decrease in fetal weights, placental weights were reduced by more than $20 \%$ by day 21 in PP13Mtreated animals (and increased $>40 \%$ in the PP13 group).

Finally, treatments with wild-type PP13, but not with its truncated mutant form, enhanced expansive circumferential remodeling of the uterine veins. These are the vessels that drain the placenta and uterine corpus such that unstressed (unpressurized) venous diameters were $>45 \%$ greater in postplacental veins. A similar effect was noted in the postmyometrial and radial vessels, but at a lower magnitude (with average increases on the order of $10-20 \%)$.

Taken together, these findings are provocative as they implicate PP13 in a number of physiological processes during gestation, specifically, regulation of systemic blood pressure, vascular remodeling, and fetal-placental growth and development.

In our previous study [30], we have demonstrated arterial vasodilation and expansion. Here we focused on venous expansion. It, therefore, appears that prolonged exposure to PP13 modifies the entire uteroplacental vasculature (arteries and veins). How exactly this expansion develops requires further analysis.

These effects become even more intriguing when one considers that PP13 protein is only found in advanced mammals (primates), where it was found to have effects on placentation, and is not thought to be present in rats [18]. Our results indicate that this molecule (in both its natural and mutated form) is still capable of influencing both the cardiovascular and fetoplacental structure and

PP13 Administration to Pregnant Rats function when administered from an exogenous source, in this case miniosmotic pumps. Certainly, this is not uncommon, as most medicines are synthetic and not 'physiological', and do not exist in vivo, although their effects may be profound, and are known to target specific cellular and molecular mechanisms. Similarly, endogenous molecules may respond to exogenous stimuli, e.g. in the immune system, which has a broad receptor repertoire to various antigens that the human body has never been exposed to previously [33]. In this regard, PP13 is thought to have an effect which can be exerted through the immune actions as well, as discussed below.

Another interesting aspect of our results involves the differential responsiveness to the mutant form of the molecule, which mimicked the hypotensive effect of wildtype PP13, but not its effects on pup and placental growth or venous structure. According to Visegrady et al. [34], the carbohydrate recognition domain of PP13 consists of 8 amino acid residues, of which 4 (those on positions 53, 65,72 and 75) form a 'pocket' that fits the sugar residue in a 'key and lock' manner. An additional 4 (located on positions 55, 57, 63 and 77) add to the affinity to sugar residues [32]. The DelT 221 mutant lacks only 2 amino acid residues (on positions 72 and 75) of the 8 that are involved in enabling carbohydrate binding. However, this naturally occurring mutant molecule [29] was demonstrated by Sammar et al. [32] to be so misfolded in order to completely lack the sugar-binding capacity. Therefore, very strict conformational requirements appear to exist for mediating some of the biological actions of PP13 on vascular, placental and fetal tissues.

Than et al. [18] have found that PP13 renders the placenta immune-tolerant to the migrating trophoblasts that are carrying foreign (paternal) genes. Kaufmann et al. [35] and many others have demonstrated the importance of trophoblast invasion in spiral artery remodeling and early placentation, and an attenuation of this process is commonly thought to be important in the development of early-onset preeclampsia and intrauterine growth restriction.

Than et al. [18] also found that the truncated PP13 loses its ability to remodel the arteries or to render the placenta immune-tolerant, and Kliman et al. [36] have found that placentally derived PP13 is routed to the smallest veins closest to the placenta, where it may penetrate the venous wall via its lysophospholipase activity $[22,23]$ and aggregates/deposits near it in the decidua [36]. The effects on the venous diameter observed in this study in response to the wild-type galectin/PP13 may be carried out by means of its binding to the venous endothelium,

Fetal Diagn Ther 2016;39:56-63 DOI: $10.1159 / 000381914$ 
presumably via sugar residues. Other studies have shown that $\beta$-actin and annexin 2A have the largest affinity for PP13 [37], although, as already noted above, we do not know whether the underlying mechanisms are related to carbohydrate binding or to secondary effects due to an altered tertiary structure.

In summary, the observations reported herein are provocative in their demonstration of several novel cardiovascular and reproductive actions of PP13. Since PP13 levels are reduced during early pregnancy in women destined to develop preeclampsia, additional studies that explore the structure-function relationship underlying the biological actions of PP13 and the possible therapeutic benefits in preeclamptic women (or in animal models of preeclampsia or intrauterine growth restriction, at least initially) appear to be well warranted.

\section{Acknowledgements}

The authors thank Shannon Kostin, Nicole Maille and Darren Clas for their skillful technical assistance as well as Dr. Ruth Cohen and Dr. Alla Trahtenherts at Hy Laboratories (Rehovot, Israel) for supplying fresh PP13 and mutated PP13 for the study using resources funded by the ASPRE project of the EC 7th Framework Project No. 601852. The authors also thank the Foundation of Helgu Jónsdóttur and Sigurliða Kristjánsson (Iceland) and Hananja EHF (Iceland) for financial support.

\section{References}

-1 ACOG practice bulletin. Diagnosis and management of preeclampsia and eclampsia. Number 33, 2002. American College of Obstetricians and Gynecologists. ACOG Committee on Obstetric Practice. Int J Gynaecol Obstet 2002;77:67-75.

2 World Health Organization: Make Every Mother and Child Count. World Health Report, 2005. Geneva, World Health Organization, 2005.

3 Huppertz B: Placental origins of preeclampsia: challenging the current hypothesis. Hypertension 2008;51:970-975.

4 Bujold E, Roberge S, Lacasse Y, Bureau M, Audibert F, Marcoux S, Forest JC, Giguère Y: Prevention of preeclampsia and intrauterine growth restriction with aspirin started in early pregnancy: a meta-analysis. Obstet Gynecol 2010;116:402-414.

5 Sibai BM: Diagnosis and management of gestational hypertension and preeclampsia. Obstet Gynecol 2003;102:181-192.

-6 Thadhani R, Kisner T, Hagmann H, Bossung V, Noack S, Schaarschmidt W, Jank A, Kribs A, Cornely OA, Kreyssig C, Hemphill L, Rigby AC, Khedkar S, Lindner TH, Mallmann P, Stepan H, Karumanchi SA, Benzing T: Pilot study of extracorporeal removal of soluble fms-like tyrosine kinase 1 in preeclampsia. Circulation 2011;124:940-950.

7 Mitchell B: One Birth Enabling Another Treatment of Preeclampsia Using Placental expanded (PLX) Cells. SGI Summit, Jerusalem, 2013.

-8 Cerdeira AS, Kopcow HD, Karumanchi SA: Regulatory T cells in preeclampsia: some answers, more questions? Am J Pathol 2012;181: 1900-1902.

-9 Wang K, Ahmad S, Cai M, Rennie J, Fujisawa T, Crispi F, Baily J, Miller MR, Cudmore M, Hadoke PWF, Wang R, Gratacós E, Buhimschi IA, Buhimschi CA, Ahmed A: Dysregula- tion of hydrogen sulfide producing enzyme cystathionine $\gamma$-lyase contributes to maternal hypertension and placental abnormalities in preeclampsia. Circulation 2013;127:25142522.

10 Huppertz B: IFPA Award in Placentology Lecture: biology of the placental syncytiotrophoblast - myths and facts. Placenta 2010; 31(suppl):S75-S81.

11 Redman CWG, Tannetta DS, Dragovic RA, Gardiner C, Southcombe JH, Collett GP, Sargent IL: Review: Does size matter? Placental debris and the pathophysiology of pre-eclampsia. Placenta 2012;33(suppl):S48-S54.

12 Redman CW, Sargent IL: Circulating microparticles in normal pregnancy and preeclampsia. Placenta 2008:29(suppl A):S73S77.

13 Huppertz B, Meiri H, Gizurarson S, Osol G, Sammar M: Placental protein 13 (PP13): a new biological target shifting individualized risk assessment to personalized drug design combating pre-eclampsia. Hum Reprod Update 2013:19;391-405.

14 Cetin I, Huppertz B, Burton G, Cuckle H, Gonen R, Lapaire O, Mandia L, Nicolaides K, Redman C, Soothill P, Spencer K, Thilaganathan B, Williams D, Meiri H: Pregenesis preeclampsia markers consensus meeting: what do we require from markers, risk assessment and model systems to tailor preventive strategies? Placenta 2011;32(suppl 1):S4-S16.

15 Akolekar R, Syngelaki A, Poon L, Wright D, Nicolaides KH: Competing risks model in early screening for preeclampsia by biophysical and biochemical markers. Fetal Diagn Ther 2013;33:8-15.

-16 Than NG, Romero R, Kim CJ, McGown MR, Papp Z, Wildman DE: Galectins: guardians of eutherian pregnancy at the maternal-fetal interface. Trends Endocrinol Metab 2012;23: 23-31.
17 Worthelbrew EJ, Koster MP, Cuckle HS, Stoutenbeek PH, Schielen PC, Visser GH: First-trimester placental protein 13 and placental growth factor: markers for identification of women destined to develop early-onset pre-eclampsia. BJOG 2010;117:13841389.

18 Than NG, Romero R, Goodman M, Weckle A, Xing J, Dong Z, Xu Y, Tarquini F, Szilagyi A, Gal P, Hou Z, Tarca AL, et al: A primate subfamily of galectins expressed at the maternal-fetal interface that promote immune cell death. Proc Natl Acad Sci USA 2009;106: 9731-9736.

19 Sammar M, Nisemblat S, Fleischfarb Z, Golan A, Sadan O, Meiri H, Huppertz B, Gonen R: Placenta-bound and body fluid PP13 and its mRNA in normal pregnancy compared to preeclampsia, HELLP and preterm delivery. Placenta 2011;32(suppl):S30-S36.

20 Huppertz B, Sammar M, Chefetz I, Neumaier-Wagner P, Bartz C, Meiri H: Longitudinal determination of serum placental protein 13 during development of preeclampsia. Fetal Diagn Ther 2008;24:230-236.

21 McNamara JM, Kay HH: Placental hormones: physiology, disease, and prenatal diagnosis; in Kay HH, Nelson DM, Wang Y (eds): The Placenta. From Development to Disease. Oxford, Wiley-Blackwell, 2011, chapt 8.

22 Burger O, Pick E, Zwickel J, Klayman M, Meiri H, Slotky R, Mandel S, Rabinovitch L, Paltieli Y, Admon A, Gonen R: Placental protein 13 (PP-13): effects on cultured trophoblasts, and its detection in human body fluids in normal and pathological pregnancies. Placenta 2004;25:608-622.

23 Than NG, Pick E, Bellyei S, Szigeti A, Burger O, Berente Z, Janaky T, Boronkai A, Kliman H, Meiri H, Bohn H, Than GN, Sumegi B: Functional analyses of placental protein 13/galectin-13. Eur J Biochem 2004;271:1065-1078. 
24 Redman CW, Sargent IL: Latest advances in understanding preeclampsia. Science 2005; 308:1592-1594.

-25 Balogh A, Pozsgay J, Matkó J, Dong Z, Kim CJ, Várkonyi T, Sammar M, Rigó J, Meiri H, Romero R, Papp Z, Than NG: Placental protein 13 (PP13/Galectin-13) undergoes lipid raft-associated subcellular redistribution in the syncytiotrophoblast in preterm preeclampsia and HELLP syndrome. Am J Obstet Gynecol 2011;205:156.e1-e14.

-26 Gonen R, Shahar R, Grimpel YI, Chefetz I, Sammar M, Meiri H, Gibor Y: Placental protein 13 as an early marker for pre-eclampsia: a prospective longitudinal study. BJOG 2008; 115:1465-1472.

-27 McDonald SD, Malinowski A, Zhou Q, Yusuf S, Devereaux PJ: Cardiovascular sequelae of pre-eclampsia/eclampsia: a systematic review and meta-analyses. Am Heart J 2008;156: 918-930.

-28 Farina A, Zucchini C, Sekizawa A, Purwosunu Y, de Sanctis P, Santarsiero G, Rizzo N, Morano D, Okai T: Performance of messenger RNAs circulating in maternal blood in the prediction of preeclampsia at 10-14 weeks. Am J Obstet Gynecol 2010;203:e1-e6.
29 Gebhardt S, Bruiners N, Hillerman R: A novel exonic variant (221delT) in the LGALS13 gene encoding placental protein 13 (PP13) is associated with preterm labour in a low risk population. J Reprod Immunol 2009;82:166173.

30 Gizurarson S, Huppertz B, Osol G, Skarphedinsson JO, Mandala M, Meiri H: Effects of placental protein 13 on the cardiovascular system in gravid and non-gravid rodents. Fetal Diagn Ther 2013;33:257-264.

31 Than NG, Sumegi B, Than GN, Berente Z, Bohn $\mathrm{H}$ : Isolation and, sequence analysis of a cDNA encoding human placental tissue protein 13 (PP-13), a new lysophospholipase, homologue of human eosinophil Charcot-Leyden Crystal protein. Placenta 1999;20:703710 .

32 Sammar M, Nisamblatt S, Gonen R, Huppertz B, Gizurarson S, Osol G, Meiri H: The role of the carbohydrate recognition domain of placental protein 13 (PP13) in pregnancy evaluated with recombinant PP13 and the DelT 221 PP13 variant. PLoS One 2014;9:e102832.

33 Camara NOS, Lepique AP, Basso AS: Lymphocyte differentiation and effector functions. Clin Dev Immunol 2012;2012:510603.
34 Visegrady B, Than NG, Kilar F, Sümegi B, Than GN, Bohn H: Homology modelling and molecular dynamics studies of human placental tissue protein 13 (galectin-13). Protein Eng 2001;14:875-880.

35 Kaufmann P, Black S, Huppertz B: Endovascular trophoblast invasion: implications for the pathogenesis of intrauterine growth retardation and preeclampsia. Biol Reprod 2003; 69:1-7.

-36 Kliman HJ, Sammar M, Grimpel Y-I, Lynch SK, Milano KM, Pick E, Bejar J, Arad A, Lee JJ, Meiri H, Gonen R: Placental protein 13 and decidual zones of necrosis: an immunologic diversion that may be linked to preeclampsia. Reprod Sci 2012;19:16-30.

37 Than NG, Romero R, Meiri H, Erez O, Tarquini F, Barna L, Szilágyi A, Ackerman R, Sammar M, Füle T, Karászi K, Kovalszky I, Dong Z, Kim CJ, Závodszky P, Papp Z, Gonen R: PP13, maternal ABO blood groups and the risk assessment of pregnancy complications. PLoS One 2011;6:e21564.

38 Than NG, Balogh A, Romero R, Kárpáti É, Erez O, Szilágyi A, Kovalszky I, Sammar M, Gizurarson S, Matkó J, Závodszky P, Papp Z, Meiri H: Placental protein 13 (PP13) - a placental immunoregulatory galectin protecting pregnancy. Front Immunol 2014;5:348. 